\title{
Afro-Ethnic Hairstyling Trends, Risks, and Recommendations
}

\author{
Sara Asbeck*, Chelsi Riley-Prescott, Ella Glaser and Antonella Tosti
}

Citation: Asbeck, S.; Riley-Prescott, C.; Glaser, E.; Tosti, A. Afro-Ethnic Hairstyling Trends, Risks, and Recommendations. Cosmetics 2022, 9, 17. https://doi.org/10.3390/ cosmetics 9010017

Academic Editor: Enzo Berardesca

Received: 12 December 2021

Accepted: 19 January 2022

Published: 26 January 2022

Publisher's Note: MDPI stays neutral with regard to jurisdictional claims in published maps and institutional affiliations.

Copyright: (C) 2022 by the authors. Licensee MDPI, Basel, Switzerland. This article is an open access article distributed under the terms and conditions of the Creative Commons Attribution (CC BY) license (https:// creativecommons.org/licenses/by/ $4.0 /)$.
Department of Dermatology and Cutaneous Surgery, Miller School of Medicine, University of Miami Hospital, University of Miami, Miami, FL 33125, USA; car365@med.miami.edu (C.R.-P.); ella.glaser@jhsmiami.org (E.G.); ATosti@med.miami.edu (A.T.)

* Correspondence: smp268@med.miami.edu

\begin{abstract}
Hairstyling trends among Black women fluctuate with social, cultural, and environmental pressures. Dermatologists should be aware of current trends and their associated risks in order to provide the best care to this population. In order to summarize the updated trends and associated health risks for the most common hairstyles worn by Black women, a literature review was performed. PubMed and EMBASE were used to identify articles related to hair styling practices, studies on the effects or risks of various styling practices, and magazine articles citing current styling trends among women of African descent. All hairstyles were found to have associated health risks; however, natural styles had the fewest adverse associations of all styles reviewed. Central Centrifugal Cicatricial Alopecia (CCCA) is the most cited hair disorder in this population, possibly linked to both chemical relaxants and traction styles. Additional studies are needed to further establish causality between these styles and CCCA. Additionally, while acceptance of natural hairstyles is on the rise, there is more work to be done throughout society to help protect and encourage women who choose to wear Afrocentric styles. Dermatologists should be well versed in these hairstyles and ready to lend appropriate advice to patients when it is requested.
\end{abstract}

Keywords: hairstyle trends; African-ethnic hair; Afrocentric hair; chemical hair relaxing; central Centrifugal cicatricial alopecia; traction alopecia

\section{Introduction}

Hair is an essential part of a person's identity, self-perception, and can be a way they communicate with those around them. It can reflect not only someone's health but their sense of style, and can be changed often and easily to portray an individual's changing attitudes and trends. Hairstyles among women of African descent, thus, vary considerably by country, region, occupation, generation, and personal style. Today, the Black hair care industry is a half-trillion-dollar industry. In fact, Black women spend more on their hair than any other ethnic group [1]. Social and environmental pressures continue to influence what society views as beautiful and culturally acceptable. There has been substantial debate and discussion regarding the association between certain hairstyles worn by Black women and their connection to hair disorders and other health conditions common to this population. Given the underrepresentation of Black dermatologists in clinical practice, it is crucial to remain up to date on current trends in this community and consider how they may be influencing and/or potentially harming patients' dermatological as well as overall health. In this educational literature review we aim to identify and summarize the current trends, potential risks, and updated recommendations for the most commonly cited hairstyles worn by women with Afro-ethnic hair. We hope to investigate and clarify the associations between these styles and the hair and health conditions common to this population, drawing connections between the various studies included in the review. The results will be organized by subsection according to the hairstyle being discussed. 


\section{Methods}

A total of 59 articles and case reports pertaining to Black or Afro-ethnic hairstyles were included in this literature review. Articles were collected primarily through PubMed and EMBASE databases using the following key words: Black hair, Afrocentric hair, ethnic hair, Afro-ethnic hair, Afro-textured hair, African-American hair, African-American hairstyles, hairstyle trends, chemical hair relaxing, hair straightening, hair braids, cornrows, dreadlocks, protective hairstyles, and hair weave. Inclusion criteria for this review: (1) Studies or reports related to hair styling practices in women of African descent, (2) studies or reports on the effects/implications/risks of various hair styling practices in Afro-textured hair, (3) magazine articles citing current styling trends among women of African descent, and (4) articles in English or translated to English. Articles on the pediatric population, or not explicitly relevant to the topic of study were excluded.

\section{Results}

\subsection{Chemically "Relaxed" Hair \\ 3.1.1. Introduction}

Since the advent of chemical hair relaxers in the 1940s, the trend among women of African descent to achieve long, straight, socially desirable hairstyles has fluctuated, but nonetheless remained very popular. In studies discussing hair relaxer use among AfricanAmerican women, prevalence of those who endorsed having ever using relaxers ranged from $71 \%$ in a group of 1693 African-American women from Detroit [2], to $95 \%$ in the Black Women's Health Study, a prospective cohort of 59,000 self-identified Black American women [3]. Reasons cited for the use of relaxers include increased hair manageability [4], decreased work of combing [5], and improved self-esteem [6].

Hair relaxing or "perming" has generally been viewed as a way for Black women to assimilate into Eurocentric standards of beauty and professionalism. In fact, relaxer use is highest in the Southern states, a region where, historically, resistance to de-segregation and discrimination against Black individuals was very widespread [7]. Many women here may have felt increased social pressures to blend in and de-emphasize their African traits. A trend toward more natural or "Afrocentric" hairstyles briefly re-appeared in the late 1960s and 1970s, during the historic Black Power movement, when women who wore their natural hair texture were seen as a making a radical political statement [8]. This movement was short lived, however, and popular movies and television shows throughout the decades have continued to perpetuate the idea that Black hair is more beautiful when chemically altered by predominantly casting Black women with long, flowing, straight hair [9]. Additionally, in many professional contexts, having Afro-textured hair is associated with negative stereotypes and lower expectations, with Eurocentric hairstyles more frequently adopted in the workplace in order to facilitate social acceptance [6].

Despite these social pressures, the use of relaxers has been on a general decline over the past decade, as the wearing of natural hair has become more acceptable, and many have begun to realize the harmful side effects that frequent chemical exposure may cause [7]. In fact, consumer studies have demonstrated that between 2008 and 2012, the market for hair relaxers declined by twenty percent [10]. There have been small pockets of resistance to the modern natural hair movement, with Black women who tried to "go natural," only to find the process confusing and extremely time consuming [11]. Understandably, since many women have worn relaxed hair for the majority of their lives, they may find natural curly hair difficult to manage as they are not used to the hairstyling process and perhaps the additional time and products needed to be able to style and maintain their curls [8]. Still, there is a general trend toward less hair relaxer use, and more natural or "protective" hairstyles, as will be discussed in the subsequent pages.

\subsubsection{Associated Risks}

Permanent straightening of Afro-textured hair can only be accomplished through the breaking and rearranging of disulfide bonds in the hair shaft [12]. The original method of 
hair relaxing, also called lanthionization, involved ingredients such as sodium hydroxide or potassium hydroxide mixed with potato starch [12]. Modern curl relaxers can be divided into lye and no-lye formulations, with lye relaxers most common in professional hair salons, and no-lye more popular for at-home use [13]. Lye-based relaxers are found to penetrate the hair shaft more quickly, resulting in rapid straightening and reduced susceptibility to scalp dryness and irritation than the no-lye relaxers [12]. The most frequently cited risks associated with the use of hair relaxers included hair damage, skin irritation, carcinogen exposure, endocrine-disrupting chemical exposure, hair loss, and lack of exercise-friendly hairstyle options.

The chemicals used during lanthionization are extremely alkaline. Not only does this pose a risk for chemical skin burns, but when left on the hair too long, relaxers begin to digest the hair itself [14]. Additionally, relaxer use results in a decreased sulfur content of the hair, which can lead to weakening, increased fragility, hair breakage, and thinning [15]. In a study of 715 African-American women aged 18 and over, $90 \%$ of women experiencing hair breakage reported using chemical treatments. Of the women who did not report breakage, they endorsed having a less involved grooming process with fewer products, fewer appliances, and longer intervals between straightening treatments, if used at all [16].

The potentially carcinogenic nature of hair relaxers is still being researched, but there have been several studies demonstrating an association between regular use of hair relaxers and tumorigenesis. Numerous chemicals present in hair relaxer formulations have been found to be carcinogenic in animal studies [10] and are hypothesized to increase cancer risk via exposure to hormonally active or endocrine-disrupting chemicals through dermal absorption [3]. Use of relaxers and hair oils have been associated with early menarche, a risk factor for breast cancer [3], and duration of relaxer use was positively associated with risk of uterine leiomyomata, also known as fibroids, another hormone-dependent disease process [17]. In a large case study of 2998 women with breast cancer, overall duration of relaxer use $>10$ years and use of relaxers before age 12 was significantly associated with larger tumor size [18]. Another study found little evidence to support an association of overall hair relaxer use with risk of breast cancer but did find an association of heavy use of lye-based relaxers with increased risk of ER+ breast cancer [3].

When women with chemically relaxed hair complain of "hair loss," they are most often describing hair breakage resulting from overprocessed and brittle hair strands [12]. There is, however, substantial debate as to the role chemical hair relaxers play in the disease processes of hair loss disorders such as Central Centrifugal Cicatricial Alopecia (CCCA), the most common type of scarring alopecia in this population [19]. This type of alopecia, formerly known as "hot comb alopecia," begins at the crown and vertex, and progresses centrifugally, leaving behind a shiny smooth scalp with follicular dropout [19]. Although the relationship between chemical relaxers and CCCA is uncertain, several case studies have been published linking the two together.

A study on 1018 patients presenting to an alopecia clinic demonstrated that patients with cicatricial alopecia were significantly more likely to report any previous chemical hair straightening than patients with non-cicatricial alopecia, and that patients with CCCA reported a significantly higher cumulative exposure to chemical straightening than controls [20]. In a prospective study by Uhlenhake et al. [19], biopsies were examined from subjects at random without clinical evidence of alopecia, but who reported use of either chemical relaxants or thermal straighteners within the past month. Interestingly, periinfundibular lymphocytic inflammation and concentric infundibular fibrosis, some of the histopathologic findings characteristic of, but not necessarily unique to CCCA, were found in all biopsies [19]. These data suggest that there may be a histological prelude to the clinical signs of CCCA and lends plausibility to the association between relaxer use and scarring alopecia. It is also important to note that some studies found no association between relaxer use and CCCA [21], or a weak, but statistically insignificant association [22], further suggesting that more studies are needed to elucidate the true relationship between the two. 
Another less commonly discussed relationship, but one that has profound health implications, is the exercise-prohibitive nature of chemically relaxed hairstyles. An estimated $80 \%$ of African-American women are overweight or obese, so the avoidance of exercise due to one's hairstyle could be a notable public health challenge [23]. In a study on 61 women of self-reported African, Afro-Caribbean, or African-American race, $18 \%$ of women stated that they exercised less than they would like because of their hair, for fear of "sweating out" hairstyles, and due to the time needed to restyle hair [24]. Another study, this time on 1558 self-identified African-American women from Detroit, MI, found that in adulthood, women who used chemical relaxers at least two times per year were $10 \%$ less likely to report intense physical activity compared to their counterparts reporting rarely/never use [25], suggesting this may be implicated as a contributor to the poor cardiometabolic health outcomes among Black/African-American women.

\subsubsection{Recommendations}

Several recommendations have been proposed in response to the research conducted on chemically relaxed hair to ensure the process is carried out in the safest and least damaging way possible. It is important for dermatologists and other health care providers to be knowledgeable of these recommendations in order to best advise their patients. First off, relaxers are best applied by experienced professionals with training in the appropriate application method and processing time [26]. The condition of the client's hair and scalp should be evaluated prior to application, and if the scalp is in any way compromised, relaxer use should be avoided, even if a base is used [15]. Thin and/or porous hair process faster and, therefore, relaxer should be left on for as little time necessary to achieve the desired results [15]. Careful application to the new growth only and deep conditioning treatments can help prevent breakage [27]. Advice for how frequently to relax hair varied from every six to eight weeks [6] to eight to 12 weeks [28], with the general theme being that if treatments are applied too frequently, the risk of hair damage and breakage is increased. Following hair relaxation, high-tension styles [29], as well as excessive combing or styling [12] should be avoided to prevent traction alopecia (TA) and excessive breakage, respectively. TA will be discussed more in depth in the section titled "Traction Hairstyles" below.

\subsection{Keratin Treatment}

\subsubsection{Introduction}

The Brazilian Keratin Treatments (BKTs) are a newer hair straightening method that has recently gained popularity due to its ability to straighten the hair with additional claims of decreasing frizz and increasing shine for up to five months [30]. The technique began in Brazil and involves applying a formaldehyde or formaldehyde-releasing solution to the hair, followed by blow drying and flat ironing to seal the straightened hair appearance [23]. The formaldehyde is necessary for the synthetic hydrolyzed keratin to properly cross-link and adhere to the natural keratin in the hair shaft [31]. Manufacturers claim that it can be safely used on chemically relaxed, highlighted, and color-treated hair [23].

This semi-permanent treatment is increasingly being used as an alternative to traditional chemical relaxers [6], and is attractive to women of African descent, as it makes naturally curly hair more manageable and frizz-free for several months at a time [30]. For many Black women, it is touted as a good option when transitioning from relaxed to natural hair, as it offers a way to camouflage new growth until the relaxed hair grows out [32]. However, as this is a relatively new hair treatment, there are very little published data on the overall popularity and frequency of use of BKTs among African-American women.

\subsubsection{Associated Risks}

Although, on the surface, Brazilian Keratin Treatments appear to be a safer alternative to chemical hair relaxing, they are not without risk. Perhaps the most concerning characteristic of BK treatments is their relatively high concentration of formaldehyde, a known 
human carcinogen [6]. Formaldehyde is known to cause respiratory irritation, contact dermatitis, headaches, pregnancy complications, and is associated with respiratory and hematopoietic malignancies at high concentrations [33]. A cohort study conducted by the National Cancer Institute found an increased incidence of lymphoproliferative malignancies, particularly myeloid leukemias, among factory workers in formaldehyde-producing and formaldehyde-using plants [30]. Although the percentage used in cosmetics is much lower than those in industrial usage, it still raises the question as to what level, if any, formaldehyde exposure can be considered "safe."

With the concern surrounding the use of formaldehyde, formaldehyde-releasing BKT formulations (marketed as formaldehyde-free) such as glyoxylic acid have grown in popularity, with no scientific proof that they are any safer than formaldehyde formulations [31]. The US Cosmetic Ingredient Review Expert Panel set a maximum safe concentration of formaldehyde at $0.2 \%$, a level that is often well surpassed by commercial BKT brands [33]. In a study done on seven different BKT brands marketed in South Africa in 2012, six had concentrations of formaldehyde five times higher than the recommended level, including five brands that were labelled "formaldehyde-free" [33]. Another study carried out in Brazil examined 23 brands, and all exceeded $0.2 \%$ formaldehyde, ranging from 18 to 54 times the permitted levels [31].

\subsubsection{Recommendations}

There is a clear concern for false labelling of Brazilian Keratin Treatments, and further regulation needs to be enforced surrounding the use and concentration of formaldehyde in these products. Many recommend avoiding these treatments altogether, due to the risks of formaldehyde and the discrepancies between listed ingredients and the actual presence of this carcinogenic ingredient in BKT preparations [31]. Additionally, during treatment, a strong odor of formaldehyde along with irritation of the eyes and oropharynx should prompt immediate suspension of treatment, as it indicates very high concentrations of formaldehyde that are likely unsafe and dangerous to health [31]. More research is needed on the health and safety implications of long-term keratin treatment use before this can be recommended to patients as a safe alternative to chemical relaxers.

\section{3. "Natural" Hair}

\subsubsection{Introduction}

Natural hairstyling refers to the discontinuation and/or avoidance of chemical relaxers to straighten the curl pattern of the hair [34]. As mentioned previously, natural hairstyles have been gaining substantial popularity over the past few decades. A study on 200 AfricanAmerican women from Detroit reported a $10 \%$ increase (26\% to 36\%) among women reporting wearing non-chemically relaxed hair between 2010 and 2011 [35]. Consumer trends research has documented a 26\% decrease in relaxer sales between 2008 and 2013, and a 17\% decrease between 2006 and 2011 [23]. Another study on 242 women of African descent from London, UK, found that natural hairstyles were actually the predominant hairstyle $(52.6 \%)$ in their study population, with respondents citing their desire for healthy hair and self-directed hair grooming as reasons for going natural [36].

With the dearth of natural hair care products currently on the market and the internet overrun with hair blogs and YouTube tutorials, "going natural" has never been easier. However, many Black women continue to face professional and social pressures to chemically straighten their hair. Even in the current times, women continue to face discrimination for wearing their natural hair texture. Additionally, natural hair may be time-consuming and difficult to maintain, especially for women who have worn straight hair their entire lives.

\subsubsection{Associated Risks}

Discrimination, and subsequent psychological damage, were the most poignant identified risks that women of African descent experience when choosing to wear their hair natural. Starting from a very young age, Black children are faced with racially biased hair 
grooming policies at their schools, and begin to experience harassment from their peers based on the appearance of their hair [37]. This continues into adulthood, where people of color are excluded from certain jobs or even sent home from work on the premise that certain hairstyles, such as dreadlocks, do not meet the company's standards of professionalism [38].

One interesting study whereby participants were asked to rate photos of Black women with different hairstyles found that employment candidates with Afrocentric hairstyles were rated as less professional and less likely to succeed in Corporate America than employment candidates with Eurocentric hairstyles [39]. One perhaps unexpected outcome of this study was the discovery that Black evaluators, compared to their White counterparts, gave higher penalties to the Black employment candidates who wore Afrocentric vs. Eurocentric hairstyles, and rated them as more dominant and less professional. This suggests that, at least in the corporate workforce, much of the discrimination towards Afrocentric or natural hairstyles may be from Black individuals themselves, who may be particularly judgmental toward traits exhibited by other Black people that are deemed to be socially unacceptable-a phenomenon called horizontal hostility [39].

Afrocentric hairstyles may be labeled as unprofessional, unkempt, and messy, and may even be considered radical or political to wear in certain environments [9]. A qualitative investigation of 90 African-American guided hair autobiographies examined the psychological significance of hair discrimination among this community, and found that emotional responses surrounding hair discrimination were present in about half of the narratives collected, with sadness and self-conscious emotions such as shame and embarrassment common among participants [37]. Participants in this study frequently resorted to chemically or thermally changing their hair texture or changing the length of their hair in response to discrimination [37].

Among the literature, the other most identified barrier among women deciding to "go natural" was unfamiliarity and or frustration with their natural tresses. As explained by one author [35], many women of African descent have had their hair chemically straightened since they were young girls and never learned to care for their hair in its natural state. For many Black women, they have had straightened hair longer than they have had natural hair, and many prefer the manageability associated with relaxed hair, or simply prefer the way straight hair looks [11].

\subsubsection{Recommendations}

Recommendations regarding caring for natural hair include washing hair with a shampoo-containing product at least every two weeks [40], detangling with detangling conditioners and a wide-tooth comb [41], and frequent moisturization with conditioning agents to prevent breakage [34]. Author Biakolo says that persistence is required to maintain natural hairstyles and cautions women that it may take time for them to become skillful at meeting the demands of their natural hair [8]. Collectively as a society, we need to continue to promote and embrace natural hairstyles both among the Black community and as allies among the majority population. As of October 2020, the CROWN (Create a Respectful and Open World for Natural Hair) Act bill, created to ensure protection against race-based hair discrimination in the workplace and in public schools, was passed by 7 states and the House of Representatives, as well as being considered for the US Senate [38]. It's continued success will depend on increasing support from allies, especially influential leaders such as health care professionals, given the underrepresentation of people of color in legislative positions [38].

\section{4. "Traction" Hairstyles: Braids, Weaves, Wigs, Twists, and Dreadlocks}

\subsubsection{Introduction}

Braids, weaves, wigs, twists, cornrows, and dreadlocks are examples of low-maintenance, chemical-free hairstyling techniques popular among women of African descent. These hairstyles typically require a time commitment upfront, ranging from hours to even days 
for more elaborate styes, but can be kept in place for long periods of time and require minimal daily effort to maintain [14]. Additionally, none of these hairstyles utilize chemical straightening, and apart from dreadlocks, are considered "protective hairstyles" because they do not require excessive daily manipulation. They can be used to help transition from chemically treated hair to natural hair, effectively masking any differences in structure or texture along the shaft of the hair [42].

Braids are one of the most versatile of the traction hairstyles. Cornrows are achieved by braiding the hair tightly along the scalp, loose braids can be done with one's own natural hair, or with the addition of synthetic or natural hairs to create the illusion of length, twists can be created by interweaving two strands rather than three, and braids or twists themselves can be further twisted around themselves and worn in numerous patterns, styles, and up-dos [13]. They are especially exercise-friendly, and can be shampooed regularly, as exposure to water or moisture will not alter the style [43]. The use of braids is popular among women with Afrocentric hair and is often seen as an expression of Black culture and style, commonly worn by African-American entertainers such as Beyoncé, and sports stars such as Venus and Serena Williams [14].

Hair weaves involve parting and cornrowing natural hair along the scalp, followed by sewing, braiding, clipping, or gluing hair extensions into the cornrow [14]. Women of all ethnicities use hair extensions or weaves, but they are particularly popular among Black women, as they can be used to simulate longer, fuller, and/or straighter hair [43]. Hair extensions can be made from either human or synthetic hair and are more resistant to moisture than natural straightened hair [43]. Weaves typically last around two months, and can be very convenient for wearers, as their hair and scalp are protected but easily accessible for washing, conditioning, and moisturizing [6]. Wigs are similar to weaves in that they are a way to cover one's natural texture and simulate length but are much less permanent as they can be removed at any point in time. They are often worn over short hair or cornrowed hair as a quick and easy protective style, decreasing the amount of manipulation and damage to the underlying hair [34].

Dreadlocks and sisterlocks involve sectioning natural hair and palm rolling, twisting, or using tools such as crochet hooks to "lock" the hair [23]. "Dreads" or "locs" have a long history within Jamaican Rastafarian culture, but are quite popular among all Black men and women today, as they are extremely low-maintenance and easy to maintain for a long period of time [23]. They are typically considered permanent until the decision is made to cut them off, but recent techniques have also been developed to untwist and pick out locks using water and conditioners [23]. Faux-locs are another option where synthetic hair is tightly wrapped around natural hair strands to create the illusion of dreadlocks without the same level of commitment. They are exercise friendly and offer another chemical-free alternative to daily styling.

\subsubsection{Associated Risks}

Common to all the aforementioned "traction hairstyles" is the risk for traction alopecia. Traction alopecia (TA) is hair loss secondary to tension placed on the follicles, often appearing as thinning along the hairline with preservation of hair at the anterior border, termed the "fringe sign" [23]. Any hairstyle that involves continuous pulling of the hair in one direction can result in significant breakage and TA, and when combined with additional hair extensions as are commonly used in braids, twists, and weaves, can add significant extra weight to the hair follicle [34]. In a 2008 cohort study on 574 girls and 604 women from South Africa, it was found that the highest risk of TA occurred when traction hairstyles were performed on chemically relaxed hair [44]. Additionally, TA prevalence was highest with traction from artificial extensions compared with that from natural long hair (dreadlocks), suggesting that artificial extensions may be more damaging to the hair follicle, perhaps due to their additional weight [44]. Mechanical insult from excessive braiding (>eight times/year) was associated with the highest marginal traction alopecia severity scores in a small cohort study done in Johannesburg, South Africa [42]. 
The use of bonding glues for the attachment of weaves to the scalp carries additional risks as well. These adhesives use ingredients such as latex and polyacrylates, which have been associated with allergic contact dermatitis [34]. One case report described a case of a severe anaphylactic reaction that resulted from the use of hair glue containing natural rubber latex [45]. In addition, the removal of these bonding glues is known to cause hair breakage and damage, which may further contribute to hair loss and traction alopecia [34]. Another risk is that of seborrheic dermatitis. Dadzie et al. found that on average, braid or weave hairstyles are worn for 63 days, and time between washes ranged from 18 to 32 days [36]. This is in contrast with the 14-day average between washes for Black women with natural non-traction hairstyles. The infrequent shampooing and/or difficulty shampooing around braids, weaves, and twists can increase the risk for or exacerbate existing seborrheic dermatitis [14,40].

Another possible association to be aware of is that of Central Centrifugal Cicatricial Alopecia (CCCA) with traction hairstyles. CCCA was mentioned previously in the section on chemical relaxers, but has also been found to be associated with wearing braids, cornrows, and sewn-in or glued-in weaves for cumulative years [23]. It is believed that hair styles causing traction can clinically produce folliculitis of the scalp (colloquially called "hair bumps"), which can cause scarring if the inflammation is prolonged [22]. A study by Gathers et al. on women diagnosed with scarring alopecia and matched controls found that for every 10-year increment that a woman wore sewn-in weaves at least once, she was 2.8 times more likely to develop CCCA $(p=0.003)$, and for every 10-year increment that a woman wore glued-in weaves, she was 2.2 times more likely to develop CCCA $(p=0.02)$ [21]. Another cross-sectional study on 326 African-American women from Cleveland, Ohio found that the presence of diabetes mellitus type $2(p=0.01)$, bacterial skin infections $(p=0.045)$, and hair styles associated with traction $(p=0.02)$ were all significantly higher in those diagnosed with CCCA [22]. One study, whereby Khumalo et al. reanalyzed data from a previous study on traction alopecia, found no association between traction hairstyles and CCCA, but acknowledged that they were limited by the small number of participants with CCCA included in the study $(n=16)$ [46].

\subsubsection{Recommendations}

With all these considerations in mind, recommendations have been suggested for how women may decrease their risk for complications when wearing traction hairstyles. Haskin et al. [34] suggests loosening the application of braids, especially along the hairline, opting for larger diameter braids and dreadlocks to better distribute the weight placed on each individual hair fiber, and leaving braids in for no longer than two to three months. Mayo et al. [29] recommends leaving edges out when getting hair braided and instead using edge-control gel to style edges, as well as avoiding tight braided up-dos or excessively long braids, and loosening braids if pain or stinging is felt at the time of placement. Other recommendations include avoiding the use of tension hairstyles on chemically relaxed hair [23], opting for looser twists rather than braids when possible [6], washing hair with shampoo at least every two weeks no matter what style is being used [40], and avoiding bonding glue in the application of weaves, opting for sewn-in options instead [29].

\section{Discussion}

The use of relaxers has been on a general decline, but it is still quite popular among African-American women. The association between relaxer use and breast cancer is possibly the most concerning of all the health risks identified in our review. The majority of the studies utilized in our review had some association if not to an overall increased risk for breast cancer, than to a larger tumor size or a higher risk for a subtype of breast cancer. This remains a topic of debate and further studies will be needed to solidify this relationship and establish causality. The most pressing dermatological risk associated with relaxer use is CCCA. All but one study on the topic reported an association between relaxer use 
and CCCA, but further studies with greater sample sizes will be needed to solidify this relationship as well.

There is a clear concern for false labelling of Brazilian Keratin Treatments, and further regulation needs to be enforced surrounding the use and concentration of formaldehyde in these products. It is probably best to avoid these treatments altogether, due to the risks of formaldehyde and the discrepancies between listed ingredients and the actual presence of this carcinogenic ingredient in BKT preparations. More research is needed on the health and safety implications of long-term keratin treatment use before this can be recommended to patients as a safe alternative to chemical relaxers.

Traction hairstyles, although considered "protective" and low-maintenance, are not without risk. They carry increased risks of traction alopecia, and cumulative use was also found to be associated with the development of CCCA. Of all traction hairstyles, those without the use of artificial extensions (natural braided hair, twists, or dreadlocks) carry a lower risk of traction alopecia and should be recommended above those with the addition of heavy artificial hair additions.

Natural hairstyling carries with it numerous health benefits. Natural hair may lead to decreased risks of cancer via reduced chemical exposure, and when heavy or excessively tight protective styles are avoided, damage to the scalp and hair follicles is more easily prevented. Women with natural hair are also more likely to engage in physical activity, as they are able to wash and re-style their hair more easily at home, without incurring a significant expense while doing so. While it may be difficult for someone who is new to styling their hair naturally to find what works best for them in terms of daily management, an abundance of user-friendly resources and tutorials are available both on the internet and in books world-wide. Discrimination is the most poignant identified barrier that Black women face when choosing to wear their hair natural, and dermatologists need to be aware of this so that they may approach the topic with care, and also to be aware of ways they may be able to make a lasting change, for example in supporting and promoting legislation such as the CROWN act.

\section{Conclusions}

Although each style discussed comes with an associated risk (summarized in Table 1), it appears that natural hair carries the fewest adverse health effects of all the hairstyles included in our review. Dermatologists should continue to recommend natural hairstyles to their patients, especially to those struggling with TA, CCCA, or seborrheic dermatitis; however, they need to be aware of the potential psychological implications and discrimination that patients may face when choosing to wear natural hairstyles. The recommendation to choose natural hairstyles should be made gently and with sensitivity, recognizing that it is not always as easy to make the switch as it may seem. While acceptance of these natural hairstyles is on the rise, there is more work to be done throughout social and legal spheres to help protect women making not only a personal style choice, but a health-conscious one.

Table 1. Health risks associated with various hairstyles.

\begin{tabular}{|c|c|}
\hline Hairstyle & Associated Risks \\
\hline Chemically Relaxed & $\begin{array}{l}\text { Hair damage, skin irritation, carcinogen exposure, } \\
\text { endocrine-disrupting chemical exposure, CCCA, exercise } \\
\text { avoidance }\end{array}$ \\
\hline Brazilian Keratin Treatment & $\begin{array}{c}\text { Carcinogen exposure, respiratory irritation, contact dermatitis } \\
\text { headaches, pregnancy complications }\end{array}$ \\
\hline Natural Hair & Psychological damage, discrimination, frustration with styling \\
\hline $\begin{array}{c}\text { Braids, Weaves, Wigs, Twists, } \\
\text { Dreadlocks }\end{array}$ & $\begin{array}{l}\text { Traction alopecia, allergic contact dermatitis (weaves), hair } \\
\text { breakage and damage, seborrheic dermatitis, CCCA }\end{array}$ \\
\hline
\end{tabular}


Central Centrifugal Cicatricial Alopecia (CCCA) remains one of the greatest dermatological threats among the hairstyles included in our review, being found in both users of chemical relaxants and those who opt instead for longer protective styles, such as braids and weaves. However, while CCCA is widely described in this population, we still lack substantial research on this topic, with some studies supporting its connection to traction and chemical styles, and some finding no association. More studies with larger sample sizes need to be conducted in order to further elucidate the risk factors and origins of this still very mysterious disease process.

Author Contributions: Conceptualization, S.A. and A.T.; methodology, S.A. and C.R.-P.; writing, S.A.; review and editing, C.R.-P. and E.G.; supervision, E.G. and A.T. All authors have read and agreed to the published version of the manuscript.

Funding: This research received no external funding.

Institutional Review Board Statement: Not applicable.

Informed Consent Statement: Not applicable.

Conflicts of Interest: The authors declare no conflict of interest.

\section{References}

1. James-Todd, T. Hormonal activity in commonly used Black hair care products: Evaluating hormone disruption as a plausible contribution to health disparities. J. Expo. Sci. Env. Epidemiol. 2021, 31, 476-486. [CrossRef] [PubMed]

2. Gaston, S.A. Chemical/straightening and other hair product usage during childhood, adolescence, and adulthood among African American women: Potential implications for health. J. Expo. Sci. Env. Epidemiol. 2020, 30, 86-96. [CrossRef] [PubMed]

3. Coogan, P.F. Hair product use and breast cancer incidence in the Black Women's Health Study. Carcinogenesis 2021, 42, 924-930. [CrossRef] [PubMed]

4. Billero, V.; Miteva, M. Traction alopecia: The root of the problem. Clin. Cosmet. Investig. Derm. 2018, 11, 149-159. [CrossRef] [PubMed]

5. Bloch, L.D. Chemical and physical treatments damage Caucasian and Afro-ethnic hair fibre: Analytical and image assays. J. Eur. Acad. Derm. Venereol. 2019, 33, 2158-2167. [CrossRef] [PubMed]

6. Aryiku, S.A. Clinical and anthropological perspectives on chemical relaxing of afro-textured hair. J. Eur. Acad. Derm. Venereol. 2015, 29, 1689-1695. [CrossRef] [PubMed]

7. Omosigho, U.R. Changing practices of hair relaxer use among black women in the United States. Int. J. Derm. 2018, 57, e4-e5. [CrossRef]

8. Biakolo, K. Why Some Black Women Are Going Back to Relaxers; Allure; Condé Nast: New York, NY, USA, 2021.

9. Dawson, G.A.; Karl, K.A.; Peluchette, J.V. Hair Matters: Toward Understanding Natural Black Hair Bias in the Workplace. J. Leadersh. Organ. Stud. 2019, 26, 389-401. [CrossRef]

10. Helm, J.S. Measurement of endocrine disrupting and asthma-associated chemicals in hair products used by Black women. Environ. Res. 2018, 165, 448-458. [CrossRef]

11. Sandeen, D. The Reverse Transitioner: Natural Back to Relaxed; Byrdie; Dotdash Meredith: New York, NY, USA, 2020.

12. Camacho-Bragado, G.A. Understanding breakage in curly hair. Br. J. Derm. 2015, 173, 10-16. [CrossRef]

13. Draelos, Z.D. Understanding African American hair. Derm. Nurs. 1997, 9, 227-231.

14. Quinn, C.R.; Quinn, T.M.; Kelly, A.P. Hair care practices in African American women. Cutis 2003, 72, 280-282. [PubMed]

15. Richardson, V. Ten pearls every dermatologist should know about the appropriate use of relaxers. J. Cosmet. Derm. 2017, 16, 9-11. [CrossRef] [PubMed]

16. Bryant, H.; Porter, C.; Yang, G. Curly hair: Measured differences and contributions to breakage. Int. J. Derm. 2012, 51, 9-13. [CrossRef]

17. Wise, L.A. Hair relaxer use and risk of uterine leiomyomata in African American women. Am. J. Epidemiol. 2012, 175, 432-440. [CrossRef] [PubMed]

18. Rao, R. Associations of hair dye and relaxer use with breast tumor clinicopathologic features: Findings from the Women's circle of Health Study. Env. Res. 2021, 203, 111863. [CrossRef]

19. Uhlenhake, E.E.; Mehregan, D.M. Prospective histologic examinations in patients who practice traumatic hairstyling. Int. J. Derm. 2013, 52, 1506-1512. [CrossRef]

20. Marks, D.H. Association of cicatricial alopecia with chemical hair straightening. Br. J. Derm. 2019, 181, 1317-1319. [CrossRef]

21. Gathers, R.C. Hair grooming practices and central centrifugal cicatricial alopecia. J. Am. Acad. Derm. 2009, 60, 574-578. [CrossRef]

22. Kyei, A. Medical and environmental risk factors for the development of central centrifugal cicatricial alopecia: A population study. Arch. Derm. 2011, 147, 909-914. [CrossRef]

23. Griffin, M.; Lenzy, Y. Contemporary African American Hair Care Practices. Pract. Dermatol. 2015, 11, $26-32$. 
24. Ahn, C.S. Hair and Scalp Care in African American Women Who Exercise. JAMA Derm. 2016, 152, 579-580. [CrossRef] [PubMed]

25. Gaston, S.A. Hair Maintenance and Chemical Hair Product Usage as Barriers to Physical Activity in Childhood and Adulthood among African American Women. Int. J. Env. Res. Public Health 2020, 17, 9254. [CrossRef] [PubMed]

26. Rosenberg, L.; Wise, L.A.; Palmer, J.R. Hair-relaxer use and risk of preterm birth among African American women. Ethn. Dis. 2005, 15, 768-772.

27. Bolduc, C.; Shapiro, J. Hair care products: Waving, straightening, conditioning, and coloring. Clin. Derm. 2001, 19, 431-436. [CrossRef]

28. Tanus, A. Black women's hair: The main scalp dermatoses and aesthetic practices in women of African ethnicity. Bras. Derm. 2015, 90, 450-465. [CrossRef]

29. Mayo, T.T.; Callender, V.D. The art of prevention: It's too tight-Loosened up and let your hair down. Int. J. Womens Derm. 2021, 7, 174-179. [CrossRef]

30. Weathersby, C.; McMichael, A. Brazilian keratin hair treatment: A review. J. Cosmet. Derm. 2013, 12, 144-148. [CrossRef]

31. Barreto, T. Straight to the Point: What Do We Know So Far on Hair Straightening? Ski. Appendage Disord. 2021, 7, $265-271$. [CrossRef]

32. Sandeen, D. How Keratin Treatments Work with Black Hair; Byrdie: New York, NY, USA, 2020.

33. Maneli, M.H.; Smith, P.; Khumalo, N.P. Elevated formaldehyde concentration in "Brazilian keratin type" hair-straightening products: A cross-sectional study. J. Am. Acad. Dermatol. 2014, 70, 276-280. [CrossRef]

34. Haskin, A.; Aguh, C. All hairstyles are not created equal: What the dermatologist needs to know about black hairstyling practices and the risk of traction alopecia (TA). J. Am. Acad. Derm. 2016, 75, 606-611. [CrossRef] [PubMed]

35. Gathers, R.C.; Mahan, M.G. African American women, hair care, and health barriers. J. Clin. Aesthet. Dermatol. 2014, 7, 26-29. [PubMed]

36. Dadzie, O.E.; Salam, A. The hair grooming practices of women of African descent in London, United Kingdom: Findings of a cross-sectional study. J. Eur. Acad. Derm. Venereol. 2016, 30, 1021-1024. [CrossRef] [PubMed]

37. Mbilishaka, A.M. Don't get it twisted: Untangling the psychology of hair discrimination within Black communities. Am. $J$. Orthopsychiatry 2020, 90, 590-599. [CrossRef]

38. Lee, M.S.; Nambudiri, V.E. The CROWN act and dermatology: Taking a stand against race-based hair discrimination. J. Am. Acad. Dermatol. 2021, 84, 1181-1182. [CrossRef]

39. Opie, T.R.; Phillips, K.W. Hair penalties: The negative influence of Afrocentric hair on ratings of Black women's dominance and professionalism. Front. Psychol. 2015, 6, 1311. [CrossRef]

40. Roseborough, I.E.; McMichael, A.J. Hair care practices in African-American patients. Semin. Cutan. Med. Surg. 2009, 28, 103-108. [CrossRef]

41. Bosley, R.E.; Daveluy, S. A primer to natural hair care practices in black patients. Cutis 2015, 95, 78-80.

42. Molamodi, K. Quantifying the impact of braiding and combing on the integrity of natural African hair. Int. J. Cosmet. Sci. 2021, 43, 321-331. [CrossRef]

43. Williams, W.M.; Alleyne, R.; Henley, A.T. The Root of Physical Inactivity Among African American Women: Identifying Exercise Friendly Hairstyles. J. Natl. Black Nurses Assoc. 2017, 28, 26-31.

44. Khumalo, N.P. On the history of African hair care: More treasures await discovery. J. Cosmet. Derm. 2008, 7, 231. [CrossRef] [PubMed]

45. Burla, M.J. Anaphylactic reaction after ongoing exposure to hair glue: A novel case report. J. Emerg. Med. 2015, 48, 5-7. [CrossRef] [PubMed]

46. Khumalo, N.P.; Gumedze, F. Traction: Risk factor or coincidence in central centrifugal cicatricial alopecia? Br. J. Derm. 2012, 167, 1191-1193. [CrossRef] [PubMed] 